\title{
Emprunt lexical au français et empreinte sémantique en espagnol : entre synchronie et diachronie, une histoire d'extension
}

\section{Stéphane Oury}

\section{(2) OpenEdition}

\section{Journals}

Édition électronique

URL : https://journals.openedition.org/cher/7390

DOI : $10.4000 /$ cher.7390

ISSN : 2803-5992

Éditeur

Presses universitaires de Strasbourg

Édition imprimée

Date de publication : 7 juillet 2014

Pagination : 189-207

ISBN : 978-2-86820-569-8

ISSN : 1968-035X

\section{Référence électronique}

Stéphane Oury, «Emprunt lexical au français et empreinte sémantique en espagnol : entre synchronie et diachronie, une histoire d'extension », reCHERches [En ligne], 12 | 2014, mis en ligne le 13 décembre 2021, consulté le 15 décembre 2021. URL : http://journals.openedition.org/cher/7390 ; DOI : https:// doi.org/10.4000/cher.7390

\section{cc) (†) ()}

Ce(tte) œuvre est mise à disposition selon les termes de la Licence Creative Commons Attribution Pas d'Utilisation Commerciale - Partage dans les Mêmes Conditions 4.0 International. 


\title{
Emprunt lexical au français et empreinte sémantique en espagnol: entre synchronie et diachronie, une histoire d'extension
}

\author{
STÉPHANE OURY \\ Université de Lorraine - Metz
}

¿'emprunt au français est un phénomène attesté depuis longtemps et le Moyen-Âge avec des pics aux XVI ${ }^{\mathrm{e}}$ et XVIII ${ }^{\mathrm{e}}$ siècles (De Forest 369-413, Oury 2008: 31-57).

L'empreinte $\mathrm{du}$ français dans ces vocables est parfois phonique et concerne le signifiant audible (articulation et prosodie: beige, [fricative palatale sonore], papá, carné, [oxytons]). Elle est également parfois graphique et affecte alors le signifiant visible (cf élite [rôle différencié de l'accent écrit: du timbre à la prosodie]). Ces traces ont été étudiées dans des travaux précédents (García Yebra 1999, Oury 2011: 23-35).

Mais quid de l'empreinte de sens? L'empreinte de la langue prêteuse sur le signifié (notionnel et formel) est-elle intacte ou altérée? Le filtre de la langue réceptrice laisse-t-il, lui aussi, une trace? Le sens peut-il influencer la forme? Voilà autant de questions auxquelles nous essaierons de répondre.

Nous allons interroger ici l'empreinte en suivant une approche morphosémantique. Nous étudierons dans quelle mesure les rapprochements de sens ou au contraire l'absence de motivation sémantique peuvent conditionner la forme du lexème par changement de genre, transfert catégoriel, indiscrimination de lexies complexes... Un volet de sémantique lexicale mettra l'accent sur les notions d'extension(s) et d'évolution(s). L'ensemble suivra une progression allant de la trace endogène vers la trace

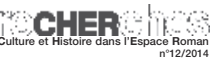


exogène et le corpus de référence sera le Diccionario de la lengua española de la Real Academia Española dans sa vingt-deuxième et dernière édition datant de 2001 (DRAE22).

\section{L'empreinte endogène: la force de l'analogie}

La langue d'accueil semble laisser elle aussi son empreinte. L'association de sens entre deux vocables sur l'axe paradigmatique des substitutions peut suffire ou contribuer à un rapprochement de genre grammatical entre les deux.

\subsection{Attraction synonymique}

L'esprit, associant deux vocables de sens voisin, associe également les genres. L'emprunt prend le genre (porte l'empreinte) du ou des synonymes préexistants: «masacre», masculin en français, devient féminin par attraction de «matanza» (féminin). "Affaire» se masculinise sous l'influence de «negocio, escándalo», "afiche» (féminin en français) adopte le genre de «cartel» (masculin), «echarpe», celui de «chal» (masculin).

\subsection{Attraction co-hyponymique ${ }^{1}$ ou sérielle}

Le changement de genre peut s'expliquer par l'appartenance du nouveau vocable à une catégorie ou série marquée au niveau du genre: c'est le cas des noms de plantes, féminins en espagnol (héritage du latin). Ainsi s'explique la féminisation en espagnol de «begonia, colza, lila, magnolia, petunia, robinia $»^{2}$.

Quant à «Entrecot, escalope», initialement féminins, ils imitent le genre de leurs co-hyponymes, majoritairement masculins: solomillo, filete...

\subsection{Attraction hypéronymique}

Les vocables adoptent le genre du vocable générique les incluant: l'hypéronyme. Ainsi «soquete», spécifique, prend le genre du vocable générique «calcetín» (masculin), «anisete» celui de "licor, alcohol» (masculins), «calés», celui de «coche» (masculin); c'est aussi le cas de «la Peugeot» devenant «el Peugeot», à l'instar de toutes les marques de voitures en espagnol.

1 Les vocables entrent dans une relation d'antonymie dans une opposition sérielle ou co-hyponymique (ils ont un hyperonyme commun, ici "plante»).

2 De plus, le «-a» est la marque du féminin en morphologie espagnole. 
Nous avons vu dans cette première partie que le vocable emprunté s'inscrit dans un nouveau système, celui de la langue réceptrice dont il va porter l'empreinte : en effet, l'élément allogène entre inévitablement dans des rapports sémantiques d'équivalence, d'inclusion qui peuvent contraindre sa morphologie.

\section{La transposition: la trace d'un transfert (catégoriel) en amont ou en aval}

Ces changements peuvent s'opérer avant l'emprunt, ici considéré en tant que processus dynamique et non résultat, l'empreinte étant française ou après l'emprunt, l'empreinte étant alors espagnole. Nous envisagerons des cas de substantivations, antonomases, souvent par ellipse, contraction d'éléments pluriels par réduction de lexies complexes.

\subsection{Le changement de catégorie grammaticale}

\subsubsection{Substantif espagnol issu d'un participe passé/ adjectif français:}

Ainsi rulé est issu de "(arrière-train bien) roulé», rapé, de "(tabac) râpé», diaprea, de "(prune) diaprée», vergé, de "(papier) vergé», cuché, de «(papier) couché », retor, de «(tissu) retors ${ }^{4} »$.

Le nouveau substantif adopte généralement le sens du groupe initial [substantif + participe] passé conduisant par là même à une restriction sémantique: "rapé» n'exprime que tabac râpé alors qu'en français il s'applique à d'autres situations (ex: fromage). L'empreinte sémantique est ici partielle: "diaprea» ne signifie que prune diaprée (irisée) alors qu'en français il s'applique souvent à une étoffe. "Retor» n'est qu'un tissu particulier en espagnol, alors qu'il peut caractériser en français un fil, un caractère. Nous pourrions inclure ici frappé, ellipse de "café frappé» dont les sens sont multiples en français mais il ne figure pas encore dans le DRAE malgré une fréquence d'emploi assez élevée.

Notons que certains changements de catégorie grammaticale ont parfois eu lieu en français, avant l'importation du vocable. Citons par exemple: «l'(armoire) commode» > «la commode» > «la cómoda» où le déterminant (adjectif qualificatif) devient substantif après ellipse du déterminé. La «livrée» a connu un itinéraire voisin: «la (tenue) livrée» est devenue «la livrée»> «la librea», par ellipse du substantif déterminé, le participe

3 Il peut également s'agir d'une étymologie erronée, J. Corominas le faisant dériver du caló «rulé» (Corominas, sv).

4 «retors» est l'ancien participe passé du verbe retordre. 
passé (déterminant) devenant du coup substantif. Nous retrouvons donc l'empreinte du français dans le genre du vocable.

\subsubsection{Le nom propre > nom commun: les antonomases}

\subsubsection{Substantif issu d'un toponyme}

\subsection{Un phénomène s'étant déjà opéré en français}

Le vocable espagnol apparaît entre guillemets, suivi du vocable français dont il est issu puis du toponyme source. Sauf indication contraire, les informations étymologiques sont tirées du dictionnaire étymologique en six volumes de Joan Corominas et J. A. Pascual. Les vocables concernés sont les suivants :

«Alepín»< alépine < Alep (ville de Syrie d'où est issue cette étoffe), "anascote» < anascot < Hondschoote (ville de Flandres où est fabriquée l'étoffe), «bauxita»< bauxite < Baux-de-Provence (ville où est extrait ce minerai), "bayoneta» < baïonnette < Bayonne, "balduque» > bolduc < Bois-le-Duc (ville où sont fabriqués ces rubans), "bistourí» < bistouri < Pistoria (ville d'Italie célèbre pour ses lames), "borgoña»< bourgogne < Bourgogne, "bujier» < bougie < Bougie (Algérie), "burdeos» < bordeaux $<$ Bordeaux, "calicó» < calicot < Calicut (ville d'Inde d'où provient cette étoffe), «camembert» < camembert < Camembert, "caolín» $<$ kaolin $<\underline{\text { Kao }}$ $\underline{\text { Ling }(\text { Chine }), ~ « c o n ̃ a c » ~<~ c o g n a c ~<~ C o g n a c, ~ « c r e t o n a » ~<~ c r e t o n n e ~}<\underline{\text { Creton }}$ (ville de Normandie où est fabriquée cette étoffe), "champán» < champagne $<$ Champagne, "chaconada» < jaconas < Jagganath (ville de l'Inde d'où

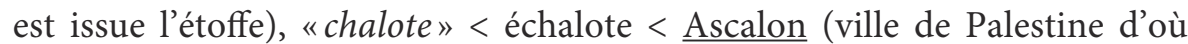
provient cet oignon), "chantillí < chantilly < Chantilly, "hacanea» < haquenée < Hackney (village d'où proviennent les chevaux), «landó» $<$ landau < Landau (ville d'Allemagne d'où est issu ce type de voiture), «manganesa» < manganèse < Magnesia (ville d'Asie d'où il provient), «martingala» < martingale < Martigues, «mayonesa» < mayonnaise $<$ Port Mahon, «muselina»< mousseline < Mossul (Iraq), "popelina» < popeline $<$ Poperhingen (ville de Flandres d'où est issue l'étoffe), «roquefort» < roquefort $<$ Roquefort, «satén» $<$ satin $<$ Tsia Toung (ville de Chine d'où provient cette étoffe), «tul» < tulle $<$ Tulle, «vichy» $<$ vichy $<$ Vichy.

Nous voyons que le vocable désignant nombre de produits, notamment des étoffes, est issu d'un toponyme, le plus souvent un nom de ville, le phénomène ayant conduit un nom propre à devenir nom commun. 


\subsection{Une particularité espagnole}

C'est le cas de "angulema» < Angoulême, berbí < Verviers (Flandres),

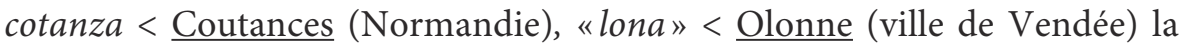
synalèphe ayant conduit à l'aphérèse $\mathrm{du} \ll \mathrm{o}$ » initial, true $<$ Troyes, lila $<$ Lille», vitre < Vitré (Bretagne). Tous ces produits sont des étoffes.

\subsubsection{Les éponymes ${ }^{6}$ ou anthroponymes}

2.1.2.2.2. Évolution remontant au français:

Le vocable espagnol apparaît entre guillemets, suivi du vocable français dont il est issu puis du patronyme source souligné. La liste est la suivante:

"Amperio» < ampère < Ampère (physicien français), «andriana» < andrienne $<$ Andrienne (du nom du personnage d'une pièce de Baron au XVIII ${ }^{\text {e }}$ siècle portant cette robe de chambre), «barbeta» < barbette < Barbe (patronne des artilleurs), «batista» < baptiste $<$ Baptiste (du nom du premier fabricant de cette étoffe), «baudio» $<$ baud $<$ Baudot (physicien français), «bechamel, besamel, besamela» < béchamel < Béchamel (financier et gourmet français), "begonia» < bégonia < Bégon (intendant français aux Antilles), "brisca» < brisque < Briscambille (comédien du XviI siècle amateur de ce jeu de cartes), «buganvilla» < bougainvillée < Bougainville (voyageur français qui a rapporté cette plante en Europe), «culombio» < coulomb $<$ Coulomb (physicien français), «chaqueta» $<$ jaquette $<$ jacques $<$ Jacques (nom donné à ce type de vêtement porté par les paysans surnommés ainsi en raison du succès de ce prénom parmi eux), "daguerrotipo»< daguerréotype $<\underline{\text { Daguerre }}$ (père de la photographie), "diamela $»^{7}<\underline{\mathrm{Du}}$ Hamel (ingénieur agronome du XviII ${ }^{\mathrm{e}}$ siècle), «damajuana» < damejeanne $<$ Dame-Jeanne (bouteille rappelant les formes très rondes d'une dame familière aux marins), «dolomía» < dolomie < Dolomieu (naturaliste français ayant découvert le minéral), «fernandina» $<$ fernandine $<$ Fernand (fabricant de cette étoffe), "guillame» < guillaume < Guillaume (inventeur de ce ciseau à bois), "guillotina» < guillotine < Guillotin (inventeur de cette machine qui a fait perdre la tête à plus d'un condamné), "hipocrás» $<$ hypocras $<\underline{\text { Hippocrate }}$ (en raison de vertus médicinales supposées de ce

5 Les toponymes suivants ne sont pas devenus des noms communs en français ou ne s'y sont pas maintenus.

6 Henriette Walter parle d'éponymes pour ces «noms communs ayant pour origine un nom propre de personne» (Walter: 75 ).

7 Ce vocable semble être une création directe de l'espagnol à partir du patronyme. Si un nom commun a existé en français, il a disparu depuis longtemps. 
breuvage), «levita» < lévite < Lévi (redingote rappelant le vêtement porté par les membre de sa tribu), «luis» < louis < Louis XIII (monnaie), «macuba» $<$ macouba $<$ Macouba (tabac cultivé par le peuple martiniquais du même nom), «magnolia» < magnolia < Magnol (botaniste français), «mansarda» $<$ mansarde < Mansart (architecte français), «napoleón» < napoléon < Napoléon (monnaie à l'effigie de l'empereur), «nicotina» < nicotine $<$ Nicot (ambassadeur français à Lisbonne ayant envoyé pour la première fois du tabac en Europe), «orfeón» < orphéon < Orphée (chœur du nom du musicien de la mythologie), "pantalón» < pantalon < $\underline{\text { Pantalone (personnage de la }}$ commedia dell'arte portant ce vêtement), «pantagruélico» < pantagruélique $<$ Pantagruel (ogre à l'appétit insatiable chez Rabelais), "pascal» < pascal $<$ Pascal (philosophe, mathématicien et physicien français), "praliné»< praliné $<$ praline $<$ maréchal Plessis Praslin (duc de Choiseul, friand de cette gourmandise), «quinqué» < quinquet $<$ Quinquet (fabricant de ce type de lampe), "robinia» < robinia < Robin (directeur du Jardin des Plantes), «sadismo» < sadisme < Sade (écrivain français), «saxofón» < saxophone < $\underline{\text { Sax }}$ (inventeur de l'instrument), «silueta» $<$ silhouette $<$ Silhouette (ministre des Finances impopulaire et souvent croqué de profil), «talma» $<$ talma $<$ Talma (tragédien français et vêtement qu'il portait)».

\subsubsection{1. Évolution propre à l'espagnol}

«Michelín» < Michelin (du nom du Bibendum aux multiples bourrelets), «leotardo»< Léotard (du nom de l'acrobate portant cette sorte de collant).

\subsubsection{Lexie complexe > lexie simple}

L'espagnol réduit parfois une lexie complexe ${ }^{8}$ (expression lexicalisée) à l'état de lexie simple ou substantif. Le canal de transmission, oral, contribue à confondre les éléments pluriels d'une lexie, notamment s'ils sont phonétiquement peu nombreux: deux, trois ou quatre syllabes, avec les syllabes d'un seul substantif. Il s'agit d'une empreinte du signifiant audible dans sa globalité au détriment du signifié. Ainsi «aide de camp, petit menu, char à banc, rendez-vous, vent d'aval, gros grain (étoffe)» deviennent-ils les substantifs suivants: «edecán, petiminí, charabán, rendibú, vendaval, gorgorán».

8 «Combinaison syntagmatique stable et autonome qui correspond à une unité de signification et intégrée en ce sens dans les dictionnaires à l'instar des lexies simples, B. Pottier parle de lexies, A. Martinet de syntèmes, Guilbert d'unités syntagmatiques» (Guerrero Ramos: 39). 
Le transfert catégoriel affecte certains vocables dans leur évolution et ne laisse d'imprimer la trace d'un état passé. L'empreinte peut être morphologique (genre) phonique (lexies indiscriminées) ou sémantique (partielle, voire en creux pour les vocables disparus en français).

\section{L'empreinte exogène: une histoire de périmètre sémantique}

\section{1. Écarts}

\subsubsection{Constat de l'écart actuel}

\subsubsection{2. Équivalence}

Dans les exemples suivants, les définitions espagnoles sont tirées du DRAE22 et les définitions françaises de Le Grand Robert de la langue française, Paris, Le Robert, 1986, 9 vol. (désormais GRLF). La forme soulignée est le vocable espagnol, la forme entre guillemets, le vocable français.

hematíe (glóbulo rojo de la sangre) / «hématie» (globule rouge du sang).

alemán (natural de Alemania, relativo a ese país) / «allemand» (de l'Allemagne).

anís (planta umbelífera) / «anis» (plante dicotylédone, Ombellifère).

3.1.1.3. Divergence partielle

chalet (1. casa de madera y tabique a estilo suizo, 2. casa de recreo o vivienda, generalmente rodeada de un pequeño jardín.) / «chalet» (1. maison de bois des pays de montagne, 2. maison de plaisance construite dans le goût des chalets suisses, 3. au Canada, maison de campagne située près d'un lac ou d'une rivière).

Somier (soporte de tela metálica, láminas de madera, etc., sobre el que se coloca el colchón) / "sommier» (1. Vx., pièce servant de support, 2. partie souple d'un lit, qui repose dans le cadre ou sur les pieds et sur lequel s'étend le matelas, 3 . gros registre.)

Vianda (1. sustento y comida de los racionales, 2. comida que se sirve a la mesa, 3. Ant., frutos y tubérculos que se sirven guisados, como el ñame, el plátano...) / «viande» (1. Vx, aliments dont se nourrit l'homme, 2 . chair des mammifères et des oiseaux que l'homme emploie pour sa nourriture, 3. Pop. et vulg., chair de l'homme, corps).

\subsubsection{Divergence totale}

Tuno (pícaro) / «thune» (aumône, pièce de cinq francs).

Bombón (1. pieza pequeña de chocolate que en su interior puede contener licor, crema u otro relleno dulce, 2. Coloq., persona 
joven y atractiva) / «bonbon» (petite friandise de consistance ferme ou dure, faite de sirop aromatisé et parfois coloré).

Acoquinar (effrayer) / «s'acoquiner» (se lier).

Adobar (cuisiner en daube) / «adouber» (armer chevalier).

$\underline{\text { Alezo }}$ (ceinture ventrale) / «alaise» (pièce de tissu parfois imperméable que l'on place sur un lit)

Balance (balancement, bilan) / «balance» (instrument qui sert à peser).

\subsection{2. Écart contemporain de la période d'emprunt}

Certaines divergences sémantiques partielles ou totales peuvent être levées au moyen d'une confrontation sémantique contemporaine de la date d'emprunt. Nous utiliserons pour ce faire des dictionnaires historiques faisant autorité, celui d'Alain Rey et celui de Joan Corominas notamment, ainsi que des dictionnaires d'édition contemporaine de la date d'emprunt: Greimas (1992a) pour l'ancien et (1992b) pour le moyen français, Furetière (1690) pour le XVII ${ }^{\mathrm{e}}$ siècle, Littré (1863-1872) pour les XVIII ${ }^{\mathrm{e}}$ et XIX ${ }^{\mathrm{e}}$ siècles, Covarrubias (1611) pour le $\mathrm{xvII}^{\mathrm{e}}$ siècle ${ }^{9}$, le Diccionario de Autoridades (1726-1739) pour le XVIII siècle, le DRAE et le Trésor de la langue française (1971-1994) pour l'époque contemporaine.

Ci-après, quelques exemples d'équivalence passée:

- Vianda (1140, Cantar de Mío Cid [Corominas, sv]) / "viande» (fin $\mathrm{XI}^{\mathrm{e}}$, par exemple in Poème de Saint Alexis [Dauzat: 791] : ensemble des aliments).

- $\underline{\text { Tuno }}$ (1765, pícaro [Corominas 573-574]) / «thune» (de 1628 à 1800, chef des gueux [Dauzat: 772]).

- Archa, archero / "arche, archer» (hallebarde, hallebardier au XII ${ }^{\mathrm{e}}$ siècle [Greimas 1992a]).

- Loyosa / «joyeuse» (XI ${ }^{\mathrm{e}}$, nom de l'épée de Charlemagne [DHLF: 1919]).

- Manijero (contremaître agricole) / «ménager»(petit propriétaire agricole, administrateur), sens vieilli (DHLF: 2190-2191).

Sargenta (religieuse)/ «sergent» (serviteur), «sens latin déjà un peu vieilli au XVI $\mathrm{I}^{\mathrm{e}}$ siècle» (DHLF: 2150) [Huguet: 16$]$.

Martel / «martel» $\left(\mathrm{xvI}^{\mathrm{e}} \text {, jalousie, souci }\right)^{10}$.

9 Les dictionnaires de début de siècle (Covarrubias, Diccionario de Autoridades) rendent compte de la langue du siècle précédent (en l'occurrence XVIe et XVIIe siècles).

10 Notons que ce sens est conservé dans l'expression «se mettre martel en tête». 


\subsection{Explication des écarts}

\subsection{1. Équivalence}

3.2.1.1. Stabilité dans les deux langues

- "Anís, festín, frambuesa, franela, lis, moda " conservent leur sens en espagnol et en français depuis le XVIII ${ }^{\mathrm{e}}$ siècle $^{11}$.

- "Chimpancé, ecarté, edredón, estiaje, evaluar, litro, marmotear, visón" sont sémantiquement stables depuis le $\mathrm{XIX}^{\mathrm{e}}$ siècle.

- Nombre d'emprunts dénominatifs récents, notamment scientifiques ou techniques gardent une signification inchangée depuis leur importation: "alucinógeno, alexia, achelense, extradós, glaciación, glucemia, glucina, glucógeno, glucosa, glucósido, glucosuria, imantación, impedancia, inductancia, infinitesimal, informática, iperita, isobaro, isógono, isómero, isonefa, isoquimena, isoyeta, percutor, resorte, tanino" ou autres: "barbotina, charada, demodé, estor, galantina, masaje, menú », par exemple.

\subsubsection{2. Évolution parallèle du français et de l'espagnol}

Il est à souligner que l'évolution est parallèle mais n'est généralement pas synchrone: la langue emprunteuse assimile les acceptions nouvelles avec un léger décalage. Ce genre d'emprunt est en fait une superposition d'emprunts: lexical d'abord puis sémantique à une ou plusieurs reprises.

\subsection{Extension:}

Obús: le passage de "mortier» à «mortier ou projectile en étant issu» a lieu en français au $\mathrm{XIX}^{\mathrm{e}}$ siècle puis en espagnol au $\mathrm{XX}^{\mathrm{e}}$.

Bagaje: "matériel d'une armée» au $\mathrm{XIII}^{\mathrm{e}}$ siècle en français, $\mathrm{XVI}^{\mathrm{e}}$ en espagnol > «objets empaquetés nécessaires au voyage» au $\mathrm{XVIII}^{\mathrm{e}}$ siècle en français, $\mathrm{XIX}^{\mathrm{e}}$ en espagnol > «ensemble de connaissances» au $\mathrm{XIX}^{\mathrm{e}}$ siècle en français, début $\mathrm{Xx}^{\mathrm{e}}$ en espagnol (Martín Fernández: 55-56).

Etiqueta: en français, l'extension métonymique de type contenant pour contenu a été la suivante: «1.écriteau, marque figurant sur un procès» au XIV siècle $>$ «2. cérémonial (figurant sur cette “étiquette")» au $\mathrm{XVII}^{\mathrm{e}}>$ «3. toute marque à visée indicative (généralisation du sens 1 )» au $\mathrm{XIX}^{\mathrm{e}}$. L'évolution a été inverse

11 Les dates sont celles de la première occurrence dans un dictionnaire de la Real Academia. Pour certains vocables, ils sont plus anciens: «anís» est par exemple déjà présent chez Nebrija (1495). 
en espagnol qui a d'abord emprunté le sens 2, sous l'influence de Carlos V, puis le sens 3. au XIX ${ }^{\mathrm{e}}$ siècle.

Usier (1739), ujier $(1580)<\mathrm{f}$. «huissier» (1138): "portier, garde de palais » > «officier de justice» $\left(\mathrm{XVI}^{\mathrm{e}}\right)$.

\subsection{Restriction:}

La restriction a parfois lieu dans les deux langues ou seulement en français mais à une date antérieure à la date d'emprunt:

- Lira $(1535)<$ anc. f. "chiere» auj. «chère»: l'espagnol emprunte au $\mathrm{XVI}^{\mathrm{e}}$ siècle, le sens de "ripaille», le sens initial de "figure» (de même racine que «cara», déjà vieilli). Le sens de «bombance» dérive de l'expression «faire bonne chère ( faire bonne figure, accueillir comme il se doit > proposer un bon repas, dans un rapport d'effet à cause [Corominas]). Nous sommes passés du visage à l'expression du visage, puis au repas qui cause cette expression.

- Flota: emprunte au français le sens d' «ensemble d'embarcations ${ }^{12}$ » dérivé d'un premier sens «foule, troupe» encore présent chez Amyot (Huguet: 72), mais déjà vieilli.

- Département, élite: ils ont cessé d'exprimer une action (action de partager, action de choisir [Huguet: 30]) pour exprimer un résultat (division, personnes choisies) au moment où ils sont empruntés respectivement aux $\mathrm{XVIII}^{\mathrm{e}}$ et $\mathrm{XX}^{\mathrm{e}}$ siècles.

- Boutique: a cessé de désigner un dépôt, un atelier, du peintre ou du statuaire par exemple, au $\mathrm{XVI}^{\mathrm{e}}$ siècle, pour désigner une échoppe en français moderne où il est emprunté au $\mathrm{xx}^{\mathrm{e}}$ siècle ${ }^{13}$.

- Estofa < anc. f. «stofe» aujourd'hui «étoffe»: "1. Matière, qualité» $>$ «2. Tissu». L'espagnol emprunte (1495) «qualité des tissus» puis «tissu».

- Peluca: "chevelure» $\mathrm{XV}^{\mathrm{e}}$ siècle en français, XVII ${ }^{\mathrm{e}}$ siècle en espagnol, d'abord sous la forme "perruca» > "chevelure postiche» $\left(\mathrm{XVI}^{\mathrm{e}}\right.$ siècle en français, $\mathrm{XVIII}^{\mathrm{e}}$ siècle en espagnol).

\subsection{Différenciation:}

- Corsé <f. «corset »: "vêtement de dessus porté par les hommes et les femmes. Le sens actuel date du XIX ${ }^{\mathrm{e}}$ siècle.» (Huguet: 49)

12 Il est amusant de noter que le français emprunte à son tour «armada» dans le sens d'«armée de mer».

13 L'espagnol va d'ailleurs être encore plus restrictif. 
Comme pour la restriction, le glissement sémantique peut s'être produit en français à une date antérieure à l'emprunt. C'est le cas des antonomases suivantes où le toponyme d'où était issu le produit lui avait déjà donné son nom en français, à la date de l'emprunt: $\underline{\text { balduque }}<$ (ruban de) Bois-le-Duc,

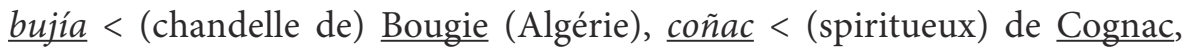
champán ou champaña < (vin de) Champagne, roquefort < (fromage de) Roquefort $\frac{14}{2}$, vichy $<$ (étoffe de) Vichy.

\subsubsection{Restriction sémantique en espagnol ou perte d'acceptions}

\subsubsection{Emprunt monosémique ou emprunt partiel}

L'espagnol n'emprunte qu'une acception du vocable français.

Fusil: le sens d'origine "pierre à feu» n'est pas pris en compte par l'espagnol qui se contente du sens dérivé "mousquet à fusil» (déterminant pour déterminé), le sens de pierre apparaît encore en français dans "fusil à aiguiser les couteaux».

Lefe: n'a retenu que l'extension métaphorique (qui est à la tête) et non le sens originel (tête) déjà vieilli au $\mathrm{XVI}^{\mathrm{e}}$ siècle mais qui s'est maintenu dans certaines expressions en français (couvre-chef, par exemple).

Román: ( $\mathrm{XIX}^{\mathrm{e}}$ siècle) est emprunté dans le seul sens de «langue dérivée du latin» puis «roman espagnol, c'est-à-dire langue espagnole» (déterminant pour déterminé), aujourd'hui c'est un archaïsme.

Les emprunts récents (1970-2001) s'inscrivent dans cette tendance à l'emprunt monosémique: affaire (seulement scandale), boîte (seulement discothèque), boutique (seulement boutique chic), chef (seulement cuisinier), debut (seulement grands débuts atistiques, sportifs), entrenar (seulement en sport), flambear (seulement en cuisine), maître (seulement maître d'hôtel)15, maillot (surtout de cycliste), mousse (alimentaire), paspartú (seulement encadrement), plató (seulement de cinéma ou télévision), sirope (en cuisine), souvenir (objet), suite (seulement chambre d'hôtel), troupe (d'artistes). On passe souvent du générique au spécifique, dans une volonté de limiter la polysémie et d'éviter une synonymie gênante. Le vocable emprunté ne semble pas entrer en concurrence avec le vocable préexistant,

14 Steve Ullmann cite précisément les trois exemples précédents comme «changements composites » : métonymie et/ou ellipse, (Ullmann : 291-292).

15 «foie gras» semble être une exception d'extension par rapport au français (foie gras + pâté de foie). 
il se différencie, se spécialise dans un domaine de compétence reconnu à la langue prêteuse (luxe, gastronomie, tourisme, scandale ${ }^{?}{ }^{16} \ldots$ ).

\subsubsection{Le cas des doublets: une double empreinte}

Ils semblent s'inscrire dans la logique de quête du monosémique ou du spécifique.

3.2.2.1.1. Des vocables monosémiques différenciés.

On préfère plusieurs vocables monosémiques à un vocable polysémique ${ }^{17}$. La démarche peut être volontaire, par choix de différenciation, ou involontaire: le même vocable, ayant parfois évolué dans son orthographe et à sa prononciation, est emprunté à des époques différentes avec des sens distincts (doublets):

- Polisón / polizón: Le "polisson» français est polysémique 1. et dérivés: gueux (1616), fripon (1680), licencieux (1695), 2. «par plaisanterie, pièce de grosse toile que les femmes ajoutaient, au XIX ${ }^{\mathrm{e}}$ siècle, pour augmenter l'ampleur des reins (1823) [DHLH: 2828]». L'espagnol choisit de différencier les vocables en même temps que les sens: "polizón» recouvre les sens 1 et dérivés, "polisón» le sens numéro 2.

- Chapel / chapeo / chapelo sont synonymes (coiffure en général): " couronne de fleurs" par exemple puis " couvre-chef» / chapó désigne un jeu au billard ainsi que l'interjection dérivée (chapeau!). Chacune des deux acceptions répond à un vocable différencié en espagnol.

- Rondel (composition poétique) / rondó composition musicale, deux sens réunis sous la même entrée en français.

- Bureo (assemblée) / buró (meuble) séparent les sens du très polysémique «bureau» français. L'espagnol adopte d'ailleurs deux sens tardifs du vocable français dont l'extension métonymique est exemplaire [étoffe (à partir du XIII e siècle)] >, (tapis de table de cette étoffe) $>$ (table recouverte de ce tapis) $>$ (pièce contenant ce meuble) $>$ (assemblée s'y réunissant) [Dauzat: 116].

- Dosel (dais) / dossier (papiers)

3.2.2.1.2. Du générique au spécifique

La démarche est la même que pour les emprunts «monosémiques»:

- Capote (générique) / capó (spécifique: automobile).

16 Les «affaires» (judiciaires) seraient-elles une spécialité française?

17 Cette démarche simplificatrice (à chaque vocable correspond une acception et une seule) n'est pas sans rappeler la volonté de l'espagnol de faire correspondre un graphème à un son. 
- Pivote (générique) / pívot (spécifique: domaine du sport).

- Tropa (générique)/ troupe (d'artistes).

- Lefe (générique) / chef (cuisine)

3.2.2.2. Extension du français

- «Petit-gris» a le sens d' «écureuil» comme petigrís en espagnol mais il a également pris celui d' «escargot» en français.

- «Bonbon» a adopté un sens populaire et argotique au pluriel en français (parties génitales par métaphore), évolution qu'il n’a pas suivie en espagnol où bombón s'est, de surcroît, différencié du français (chocolat fourré, jeune fille séduisante).

- «Pince» dans un rapport métaphorique rappelant le précédent a désigné de façon populaire la main en français et s'est partiellement différencié de pinza.

\subsubsection{Extension sémantique en espagnol}

\subsubsection{Nouvelles acceptions}

\subsection{Par similarité18}

Canguro: s'est étendu par métaphore: du marsupial portant son petit dans sa poche, on est passé à la baby-sitter portant le bébé sur ses genoux et par extension gardant n'importe quel enfant.

Ogro: dans un rapport métaphorique ce vocable désigne, à la manière $\mathrm{du}$ personnage gigantesque, irascible et cruel des contes, une personne rustre ou au mauvais caractère.

Pastel: $\quad$ cette évolution est, si l'on suit Vincent Nyckees, liée à «des causes socioculturelles» (Nyckees: 107). Huguet parle, pour sa part, $d^{\prime}$ ' influences des institutions et des coutumes» (Huguet: 16) : l'artisan qui travaille la pâte, passe avec le temps du salé au sucré, le fruit de son travail (pastel) suit la même évolution.

\subsection{Par contiguïté}

- Curalla, chaple: on glisse de l'outil vers l'action. On passe de l'outil de curaille (curage chez Greimas) à plumeau en espagnol. Il se passe la même chose pour chaple dont le sens dérive de celui de «burin de chaple» (coup violent chez Furetière) vers celui de burin, en espagnol.

18 Steve Ulmann (Ullmann: 286) regroupe les phénomènes de différenciation sémantique en figures de similarité sémantique (métaphore) ou formelle (analogie) ou de contiguïté sémantique (synecdoque et métonymie) ou formelle (contagion). 
- Angulema, potajier: le glissement s'opère de déterminant vers le déterminé: tela de Angulema > tissu, oficial potajier ("officier en charge des provisions» chez Furetière) $>$ responsable des provisions.

- Chalet: du particulier (construction en bois, de montagne) on passe à un autre particulier (maison de campagne, pavillon), le lien entre les deux étant un signifié plus général et englobant, une sorte d'hypéronyme sémantique disant le sens d' «habitation».

- Hucha: du général (coffre) on passe au particulier, destiné particulièrement aux économies, la «tirelire», puis du contenant au contenu: les économies elles-mêmes.

\subsubsection{Restriction en français}

- «Martel» / martel: le sens de «jalousie, souci » (sans doute emprunté à l'italien), présent au XVIe, ne persiste que dans l'expression «se mettre martel en tête» (DHLF: 2150).

- «Sergent»/ sargenta: le sens espagnol de «religieuse» est plus proche du sens historique (latin) «serviteur» (de Dieu) que le seul sens de «sous-officier» en français.

- «Bataille» / batalla: le sens français s'est généralisé à partir de "lutte armée», les sens historiques ${ }^{19}$ apparaissent plus clairement en espagnol: «combat, centre de l'armée (infanterie), chaque partie de l'armée, tournoi, combat d'escrime» (DRAE22).

- «Gigue» / giga: le vocable a perdu en français le sens d'instrument de musique qu'il avait à l'origine et qui s'est maintenu en espagnol.

- «Marchand» / merchante: «marchand: personne faisant un marché, vendeur ou acheteur» (Huguet: 63) s'est restreint au sens de vendeur en français. En revanche il conserve son sens large en espagnol: «el que vende o compra géneros sin tener tienda fija» (DRAE22).

- «Courage» / coraje: le sens «ensemble des sentiments que l'on a dans le cœur, au Xvi siècle (Huguet: 63), s'est par la suite limité au sentiment noble du «courage» en français alors qu'il continue d'avoir celui de la «colère» en espagnol.

- «Étape» / etapa: la remarque est la même que pour «batalla», le sens français semble plus général que les sens espagnols plus fidèles aux sens historiques et au domaine militaire qui a été rapidement le sien " 1 . dépôt de marchandises, marché» > «2. magasin de vivres et munitions d'une armée en marche» > «3. lieu où les troupes s'arrêtent pour passer la nuit » > « 4 . halte en général » > « 5 . distance à parcourir »

19 Ils apparaissent clairement au XVIe siècle et sont relevés par Huguet (Huguet: 250). 
> «6. Époque, période». Les sens 2 et 3 apparaissent plus clairement en espagnol (DRAE22).

- «Grange» / granja: «grange» a été synonyme en français de ferme, au $\mathrm{XVI}^{\mathrm{e}}$, et «il l'était encore, notamment chez Rabelais» (Huguet: 63). Il $\mathrm{y}$ a ensuite eu restriction pour ne désigner qu'une partie de celle-ci.

Il semble que les emprunts anciens et/ou polysémiques se prêtent particulièrement à la différenciation sémantique partielle, en général dérivée d'un sens premier. L'extension et la variété géographiques du monde hispanique rajoutent à ce potentiel de différenciation, nombre de vocables ayant acquis en Amérique un sens différenciéz ${ }^{20}$.

\subsubsection{Différenciation sémantique français / espagnol}

3.2.4.1. Restriction différenciée dans les deux langues: Sens $1+$ sens $2>$ seulement sens 1 en français et seulement sens 2 en espagnol

Confitura: en français « confiture» a signifié: «1. aliments préparés pour la conservation »: Rabelais parle d' «ails, d'oignons et semblable confiture» (Huguet: 233) > «2. aliments confits dans le sucre: 2.a). fruits au sirop, 2.b). pâtes de fruit, 2.c). fruits confits et 2.d). fruits cuits dans le sucre avant de se limiter à cette dernière préparation, à la moitié du XIX ${ }^{\mathrm{e}}$ siècle» (DHLF: 845). Le sens 1. est déjà vieilli au moment de l'emprunt (fin $\mathrm{XVI}^{\mathrm{e}}$ siècle, d'après Corominas), l'espagnol emprunte donc le sens 2. dans sa diversité a), b), c), d), avant de se restreindre à 2.c). «fruta confitada» (DRAE22).

Le français, pour sa part, se restreint à 2.d). et les deux vocables n'ont par conséquent plus d'acception commune, bien qu'il s'agisse de deux ramifications ou cohyponymes issu d'un même tronc ou hyperonyme (sens 2.).

\subsubsection{Glissement ou déplacement sémantique en espagnol}

3.2.4.2.1. Par contiguité (métonymie ou synecdoque ${ }^{21}$ et / ou ellipse ${ }^{22}$ ).

- de «ouate» à bata (robe de chambre, blouse): (prenda de) bata ${ }^{23}$.

20 «Tropa» a également le sens de «troupeau» pour les chevaux en Amérique.

21 «S. Ullmann, à l'instar de R. Jakobson et de la plupart des théoriciens du $\mathrm{xx}^{\mathrm{e}}$ siècle, assimile la synecdoque à la métonymie (différenciées chez Darmesteter) et estime que la différence ne se justifie pas d'un point de vue psychologique et qu'elle relève uniquement d'un point de vue logique qui n'intéresse pas selon lui la sémantique», (Nyckees: 102).

22 Ullmann parle alors de «changements composites» (Ullmann: 106).

23 C'est là une hypothèse personnelle pour expliquer le lien sémantique entre le français et l'espagnol (vêtement de / en coton > coton (bata). 
- de «mariage» à mariachi (musicien mexicain): (músico de) ${ }^{24}$ mariachi.

- de «noyau» à noyó (liqueur): (licor de) noyón ${ }^{25}$.

- de «triage» à triache (café de mauvaise qualité): (café de) triache.

- de "quai» à cai (rideau de quai): (cortina de) cai.

- de «chambre» à chambra (robe de chambre): (ropa de) chambra.

L'ellipse est souvent un facteur de différenciation partielle ou totale car le déterminant, la plupart du temps un adjectif qui est devenu un substantif et qui s'est maintenu en espagnol, pouvait caractériser nombre de déterminés en français, or il s'est souvent limité à un seul de ces déterminés, lequel a de surcroît disparu, rendant le rapport sémantique emprunteur/emprunté plus obscur encore. Le vocable conserve la forme du déterminant et le sens du déterminé:

- $\underline{\text { Rulé}<~ «(c u l ~ b i e n) » ~ r o u l e ́, ~ e n ~ f r a n c ̧ a i s ~ s e u l e m e n t ~ p a r t i c i p e ~ p a s s e ́ 26 . ~}$

- $\underline{\text { Retor }<~ «(t i s s u) ~ r e t o r s », ~ e n ~ f r a n c ̧ a i s ~ s e u l e m e n t ~ a d j e c t i f ~ t i r e ́ ~ d u ~ p a r t i c i p e ~}$ passé.

- Vergé < «(papier) vergé».

- Giroflé ou jiroflé < «(clou de) girofle».

- Diaprea < «(prune) diaprée», en français seulement participe passé (irisé).

- $\underline{\text { Cuché }}<$ (papier) couché

- Cremallera < «(fermeture à) crémaillère».

Le glissement sémantique du déterminé vers le déterminant se retrouve dans le cas d'antonomase des toponymes représentant un produit qui y est fabriqué et ayant disparu en français où ils n'ont pas acquis le statut de nom commun.

\subsection{Par similarité (la métaphore):}

ponleví: l'espagnol n'emprunte au Siècle d'Or que la métaphore inspirée du pont-levis: la chaussure à talon, à la française (sens disparu par ailleurs en français).

griñón: l'espagnol a emprunté la métaphore («grenon» moustaches en moyen français > coiffe de nonne ayant cette forme). Le vocable a disparu en français.

24 Même remarque que note précédente, musicien de mariage $>$ mariage (mariachi). Notons que cette étymologie est contestée par certains étymologistes.

25 Liqueur de noyau $>$ noyau (noyó).

26 Une autre explication pourrait être une étymologie fausse, le vocable provenant du caló et signifiant «les fesses». 
3.2.4.2.3. Par mauvaise lecture:

- de «Tunis» à tuno (pícaro, gueux): tuno < argot, roi de Thunes, nom donné au chef des vagabonds, roi des gueux en France au XVII ${ }^{e}$, par allusion au "duc de la Basse Égypte» comme s'était fait appeler le chef des gitans lorsque ses bandes arrivèrent pour la première fois à Paris en 1427 (BDELC: 563). La forme «t(h)unes» («tunis» en ancien français) n'a pas été perçue comme un toponyme mais comme un synonyme, vu le sens global de l'expression, de gueux, vagabonds, le « $\mathrm{s}$ » ayant de surcroît sans doute été interprété comme une marque de pluriel, d'où le glissement sémantique Thunes $>$ tuno.

Notons enfin que le vocable français à l'origine de l'emprunt, parfois archaïque et désuet, a pu disparaître de la lexicographie française.

\section{Conclusion}

L'itinéraire sémantique est parfois chaotique mais demeure lisible dans la très grande majorité des cas; le lien sémantique entre prêteur et prêté est évident et le relevé d'empreinte révèlent une origine française sans équivoque entre conformisation (équivalence) et innovation (différenciation).

À de rares occasions, les sens sont très différenciés ou le vocable français a disparu, de sorte qu'il est alors malaisé d'établir le rapport entre prêteur et prêté.

Pour dire un sens nouveau, les langues modernes se sont à la fois enrichies de nouveaux vocables monosémiques, cela semble être la tendance actuelle de l'espagnol, et de nouvelles acceptions, développant, par là même, la polysémie. Plus l'emprunt est reculé et plus le risque de différenciation est grand, les locuteurs ayant tendance à développer des sens figurés dérivés d'une acception première à un rythme et avec une créativité différents d'une langue à l'autre.

Le vocable français importé en espagnol sort rarement indemne de cette expérience. Rhabillé à l'espagnole (graphie et/ou prononciation), il se plie au génie de la langue, perdant parfois son genre, changeant de temps en temps de catégorie grammaticale, se délestant parfois de certaines acceptions, en acquérant souvent d'autres mais cette empreinte de l'emprunt est sans doute le prix du transfert, la taxe à acquitter à la douane et à coup sûr le gage d'une intégration meilleure et plus durable. 


\section{Bibliographie}

Corominas J., 1961, Breve Diccionario Etimológico de la Lengua Castellana, Madrid, Gredos, (BDELC).

Covarrubias Orozco S., 1993, Tesoro de la lengua castellana o española (1611), Ed. Alta Fulla, Barcelona, 1 vol.

Dauzat A, Dubois J., Mitterand H., 1990, Nouveau Dictionnaire étymologique et historique, Paris, Larousse, 6 e éd.

De Forest J.B., (oct.-déc. 1916), «Old french borrowed words in the old spanishof the twelfth and thirteenth centuries", The Romanic Review, 4, p. 369-413.

Diccionario de Autoridades (DA), 1990, Diccionario de la lengua castellana [...]. Compuesto par la Real Academia Española, Tomo I. En Madrid, en la Imprenta de Francisco del Hierro, Impresor de la Real Academia Española, Año de 1726, seis volúmenes, Madrid, 1726-1739 (I, 1726; II, 1729; III, 1732; IV, 1734; V, 1737; VI, 1739), Imprenta de Francisco de Hierro, tomos I Y II, Viuda de F. del Hierro, tomo III, Herederos, tomos IV, V, VI., reproduction en fac-similé, 3 vol., Madrid, Gredos.

Furetière A., Le dictionnaire universel, 1978, Paris, Le Robert, 3 vol., $1^{\text {re }}$ édition [1690].

García Yebra V., 1999, Diccionario de galicismos prosódicos y morfológicos, Madrid, Gredos.

Greimas A.J., 1992, Dictionnaire de l'ancien français [1979], Paris, Larousse.

Greimas A.J., Keane T.M., 1992, Dictionnaire du moyen français, Paris, Larousse.

Guerrero Ramos G., 1997, Neologismos en el español actual, Madrid, Arco Libros.

Huguet E., 1967, L'évolution du sens des mots depuis le XVI siècle, Genève, Droz.

Le Grand Robert de la langue française, 1986, Paris, Le Robert, 9 vol. (GRLF).

Littré E., 1996, Dictionnaire de la langue française, Paris, Hachette, 1863-1872, 6 vol., (supplément, 1906), Versailles.

Martín Fernández M.I., 1998, Préstamos semánticos en español, Cáceres, Universidad de Extremadura.

Nyckees V., 1998, La sémantique, Paris, Belin.

Oury S., "Le français en Espagne du Moyen Age au XIxe siècle», 2008, Colloque International de Lyon in Le français ici, ailleurs et toujours, place et fonctions $d u$ français dans les autres langues hier et aujourd'hui, 9-10 mai 2005, Lyon, Université de Lyon III, Brigitte Horiot (dir.), p. 31-57.

Oury S., «Adaptation de l'emprunt lexical: approche phonétique, phonologique et graphique de quelques gallicismes en espagnol», 2011, in Les Aspects actuels de la linguistique hispanique, Actes du Colloque International de Paris 13 des 5, 6, 7 octobre 2006, Paris, Libéro, A. Desporte (dir.) : 23-35.

Oury S., "Variantes diatopiques et néologismes: le cas des gallicismes en Amérique latine», 2010, Vues et contrevues, Actes du XIIe Colloque international de Linguistique ibéro-romane, Université de Haute Bretagne-Rennes 2, p. 24-26 septembre 2008, Limoges, Lambert Lucas, p. 431-442. 
Rey A., 1998, Dictionnaire historique de la langue française [1992], Paris, Le Robert, 3 vol. (DHLF).

Trésor de la langue française (TLF), 1994, Paris, Gallimard, 16 vol.

Ullmann S., 1969, Précis de sémantique française, Berne, A. Franck, 4e éd.

Walter H., 1997, L'aventure des mots français venus d'ailleurs, Paris, Laffont. 